\title{
Hereditary Thrombotic Thrombocytopenic Purpura in a 9-Month Old: Diagnosing and Managing an Ultra-rare Disorder
}

\author{
Kirk D. Wyatt, MD, ${ }^{*}$ Mira A. Kohorst, MD, ${ }^{*}$ Lea M. Coon, MS, LCGC, $\dagger$ \\ Rachel M. Hurley, BA, $\$$ Hendrika Anette van Dorland, PhD,\| \\ and Carola A.S. Arndt, MD*
}

\begin{abstract}
Summary: Hereditary thrombotic thrombocytopenic purpura is an ultra-rare disorder caused by biallelic mutations in the ADAMTS13 gene. Because it can be difficult to diagnose, plasma ADAMTS13 activity assessment should be considered in patients with thrombocytopenia, anemia, and schistocytes on peripheral blood smear. We present the diagnostic evaluation of a patient with hereditary thrombotic thrombocytopenic purpura. Genetic testing revealed one known pathogenic mutation and one novel mutation of ADAMTS13 classified as likely pathogenic on the basis of parental genetic testing and in silico analyses. We further discuss off-label use of prophylactic plasma-derived Factor VIII (Koate-DVI) and the benefit of rare disease registries.
\end{abstract}

Key Words: TTP, congenital TTP, hereditary TTP, thrombotic thrombocytopenic purpura

( J Pediatr Hematol Oncol 2020;00:000-000)

\section{CASE REPORT}

A 9-month-old girl presented to the emergency department with fever, cough, rhinorrhea, and poor oral intake. These symptoms were present for 2 weeks but worsened over the previous 2 days. She had a history of thrombocytopenia in the postnatal period, with a platelet count nadir of $41,000 \times 10^{9} / \mathrm{L}$, which spontaneously resolved by 3 weeks of life. On examination, she had coarse breath sounds, bilateral acute otitis media, pale and mottled skin, and delayed capillary refill. She lacked lymphadenopathy or hepatosplenomegaly. Respiratory syncytial virus testing was positive. Laboratory studies revealed normal chemistries, creatinine, and transaminases. Complete blood count $(\mathrm{CBC})$ revealed normocytic anemia, with hemoglobin of $8.1 \mathrm{~g} / \mathrm{dL}$ and mean corpuscular volume of $78.7 \mathrm{fL}$. Platelet count was $13,000 \times 10^{9} / \mathrm{L}$, and leukocyte count was $11.9 \times 10^{9} / \mathrm{L}$. Uncorrected reticulocyte count was $2.8 \%$. Lactate dehydrogenase was elevated at $685 \mathrm{U} / \mathrm{L}$, and haptoglobin was undetectable. Prothrombin time, partial thromboplastin time, and fibrinogen were normal. Out of clinical concern for sepsis, blood cultures were drawn, she was given ceftriaxone, and sent to a referral center.

At the referral center, CBC confirmed anemia and thrombocytopenia. Peripheral blood smear demonstrated schistocytes and

Received for publication December 21, 2019; accepted April 22, 2020. From the *Department of Pediatric and Adolescent Medicine, Division of Pediatric Hematology/Oncology; †Department of Laboratory Medicine and Pathology, Division of Hematopathology; † Mayo Clinic Graduate School of Biomedical Sciences; §Mayo Clinic Alix School of Medicine, Mayo Clinic, Rochester, MN; and \|Department of Hematology and Central Hematology Laboratory, Inselspital, Bern University Hospital, Bern, Switzerland.

H.A.v.D. was formerly an investigator at the Hereditary TTP Registry (www.ttpregistry.net). The remaining authors declare no conflict of interest.

Reprints: Carola A.S. Arndt, MD, 200 First Street SW, Rochester, MN 55901 (e-mail: carndt@mayo.edu).

Copyright (C) 2020 Wolters Kluwer Health, Inc. All rights reserved. no blasts (Fig. 1A). Direct antiglobulin test returned negative. She was empirically given intravenous immune globulin at a dose of $1 \mathrm{~g} / \mathrm{kg}$ for presumed autoimmune cytopenias and was transfused with platelets and packed red blood cells. Two days later, her hemoglobin had risen to $12.3 \mathrm{~g} / \mathrm{dL}$ and platelet count to $430 \times 10^{9} / \mathrm{L}$.

She was followed with serial CBCs. With viral respiratory or gastrointestinal illnesses, she developed recurrence of her thrombocytopenia. Repeat peripheral blood smear performed while the patient was well demonstrated slight schistocytes (Fig. 1B). Congenital thrombotic thrombocytopenic purpura/hereditary thrombotic thrombocytopenic purpura (hTTP) (Upshaw-Schulman syndrome) was suspected, and ADAMTS13 (a disintegrin and metalloprotease with thrombospondin type 1 motif, member 13) testing revealed activity of $<5 \%$ with no evidence of functional inhibitor, leading to a diagnosis of hTTP.

Thrombotic thrombocytopenic purpura (TTP) is characterized by deficient activity of the protease ADAMTS13 which cleaves high molecular weight von Willebrand Factor multimers into smaller, lessactive fragments. In the absence of functional ADAMTS13, highlyactive high molecular weight von Willebrand Factor multimers accumulate, leading to spontaneous microvascular thrombosis and microangiopathic hemolytic anemia. TTP can be inherited, due to mutations in the ADAMTS13 gene, or acquired, due to autoantibodies which lead to inhibition and/or clearance of ADAMTS13. ${ }^{1,2}$

The patient's thrombocytopenia in the neonatal period may have represented undiagnosed TTP, although her hemoglobin and peripheral blood smear were reported as normal at that time. After her diagnosis was established, the patient underwent screening brain magnetic resonance imaging examination, which revealed no detectable abnormality, such as subclinical infarction.

Confirmatory genetic testing of the patient and her parents revealed 2 paternally-acquired mutations in the ADAMTS13 (GRCh37, NM_139025.4) gene: c.1787C > T (p.Ala596Val) on exon 16 and c. $1392 \mathrm{C}>\mathrm{T}$ (p.Gly464 =) on exon 12, with the former known to be pathogenic ${ }^{3}$ and the latter a variant of uncertain significance (VUS). In addition, the patient had a maternally acquired c.548T > A (p.Leu183Gln) missense mutation on exon 6, which was reported by the reference laboratory (Fulgent Genetics, Temple City, CA) as a VUS.

In silico analyses were performed to obtain evidence of pathogenicity of the maternally acquired mutation. Mutational analysis using subRVIS ${ }^{4}$ (subrvis.org) identified the location of the mutation as functionally important. ADAMTS13 function is calcium-dependent, and the residues surrounding this amino acid are thought to be important for calcium binding. ${ }^{5}$ Furthermore, in silico analyses using PolyPhen 2 HumVar ${ }^{6}$ (http://genetics.bwh.harvard.edu/pph2/) and Mutation Assessor ${ }^{7}$ (http://mutationassessor.org/r3/) yielded classifications of this mutation as probably damaging. Finally, the alteration was absent from 3 large population genetics databases: 1000 Genomes (www.internationalgenome.org), ${ }^{8}$ ESP (http://evs.gs. washington.edu/EVS/), ${ }^{9}$ and gnomAD (https://gnomad.broadinstitute. org/). ${ }^{10} \mathrm{On}$ the basis of this evidence, we were able to classify the maternally acquired c.548T $>$ A (p.Leu183Gln) mutation as likely pathogenic per American College of Medical Genetics and Genomics (ACMG) criteria. ${ }^{11}$ Furthermore, communication with the Hereditary 

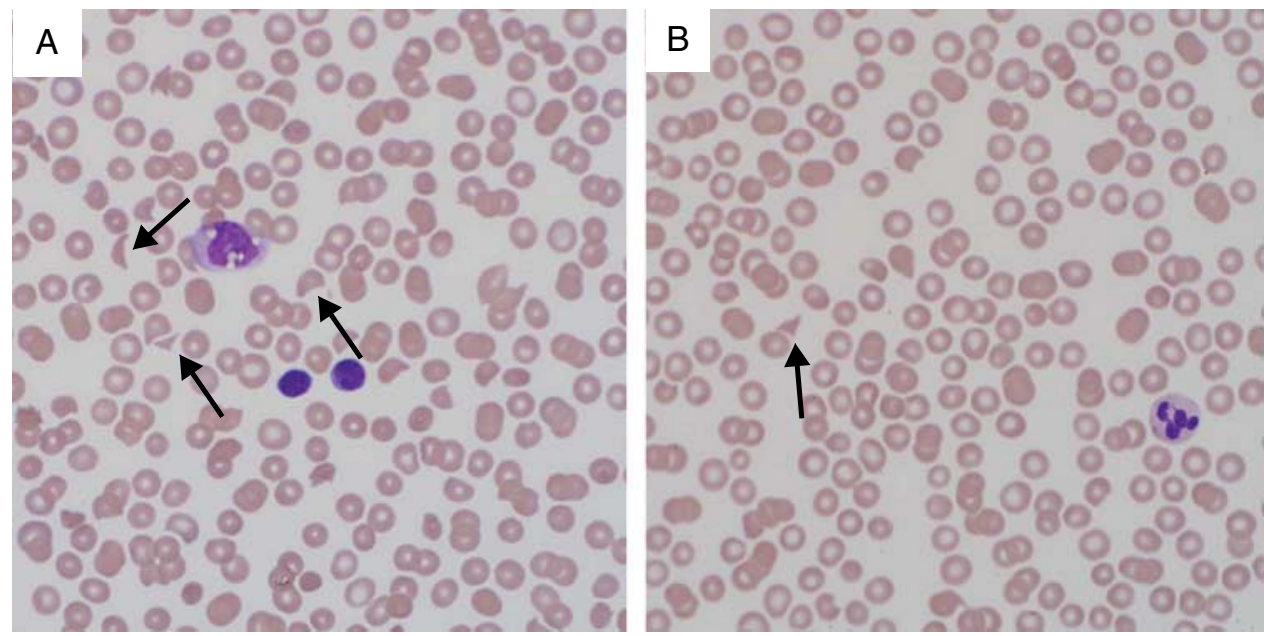

FIGURE 1. Peripheral blood smears (A) on initial presentation to the referral intensive care unit and (B) while the patient was well demonstrate schistocytes and helmet cells (arrows). full color

TTP Registry (www.ttpregistry.net) revealed a registered patient diagnosed with hTTP at 5 months of age who had a T $>$ C (p.Leu183Pro) change of the same nucleotide (c.548) that was altered $\mathrm{T}>\mathrm{A}$ in our patient. The registry patient similarly suffered from thrombocytopenia, hemolysis, and anemia in the immediate postnatal period.

\section{DISCUSSION}

hTTP is a chronic, lifelong disease with no cure. Acute episodes of anemia and thrombocytopenia are treated with infusions of fresh frozen plasma (FFP), which contains ADAMTS13, to replete the deficient protein. It is common practice to prophylactically treat patients with routine (ie, every 2 to $3 \mathrm{wk}$ ) FFP to prevent acute episodes of thrombotic microangiopathy. ${ }^{1}$ The observation that one plasma-derived Factor VIII product (Koate-DVI; Kendrion Biopharma) incidentally contains a relatively high concentration of ADAMTS $13^{12}$ has led to off-label use in patients with hTTP. ${ }^{13}$ Furthermore, recombinant ADAMTS13 is the subject of an ongoing phase III randomized cross-over clinical trial (ClinicalTrials.gov NCT03393975) comparing prophylactic versus on-demand dosing with a continuation arm. The preceding phase I study reported ADAMTS13 pharmacokinetic parameters similar to what was observed with FFP infusion. ${ }^{14}$

To gather more information about this rare disease, an international Hereditary TTP Registry ${ }^{15}$ (www.ttpregistry. net) exists to combine patient data and yield insights into the pathogenesis and management of hTTP. The patient was enrolled in the registry, which provided supplemental information to the treating team to confirm the likely pathogenicity of one of the patient's genetic VUS and also connected the treatment team with other providers who care for patients with hTTP. ${ }^{16}$

The patient was initially treated with on-demand FFP. However, she required infusions approximately every 4 weeks due to acute exacerbations. For prevention of acute TTP exacerbations, her parents elected to initiate prophylactic management with Koate-DVI to allow for the convenience of rapid, small-volume, in-home infusion and also to potentially reduce the risk of transfusion-associated viral diseases using a solvent/detergent treated product. She was

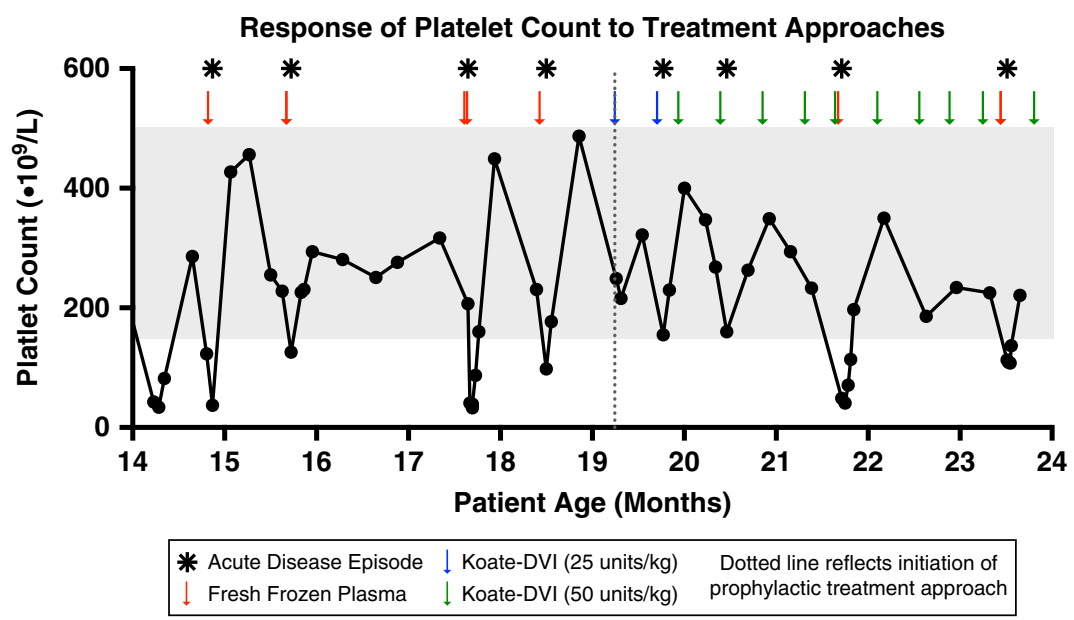

FIGURE 2. Platelet count over time. Arrows note the time of treatment administration. Acute thrombotic thrombocytopenic purpura episodes are noted with asterisks. Shaded area denotes reference range for platelet count. $\frac{\text { full color }}{0,1 i n e}$ 
initially started at a dose of $25 \mathrm{U} / \mathrm{kg}$ intravenously every 2 weeks; however, she experienced breakthrough acute TTP episodes, and her dose was modified several times before arriving at the present dose of $25 \mathrm{U} / \mathrm{kg}$ on Mondays and $40 \mathrm{U} / \mathrm{kg}$ on Thursdays. Thus far, the patient has been treated for 4 acute episodes with Koate-DVI and demonstrated improvement of anemia and thrombocytopenia within 2 days of receiving "rescue" doses (Fig. 2).

In summary, hTTP is an ultra-rare condition for which diagnosis may be delayed. ADAMTS13 assay should be considered for patients with anemia, thrombocytopenia, and schistocytes on peripheral blood smear. In this patient, persistence of schistocytes while she was clinically well was the clue which led to the diagnosis of hTTP. Patients may be treated with FFP or Koate-DVI. Patients with severe disease may require frequent dosing to prevent recurrent episodes. An ongoing trial of recombinant ADAMTS13 may lead to the availability of a new treatment for this disease in the near future. Through collaboration with a genetic counselor, we were able to establish a novel mutation as likely pathogenic, and we experienced that disease registries can be invaluable to clinicians when caring for patients with rare diseases.

\section{ACKNOWLEDGMENTS}

The authors thank the patient's family for permitting us to write this case report and share her story with the medical community. They also thank Debbie Bennes and Roeun Im for assistance obtaining peripheral blood smear photographs.

\section{REFERENCES}

1. Kremer Hovinga JA, Lammle B. Role of ADAMTS13 in the pathogenesis, diagnosis, and treatment of thrombotic thrombocytopenic purpura. Hematology Am Soc Hematol Educ Program. 2012;2012:610-616.

2. Wilson DB. Acquired platelet defects. In: Nathan DG, Oski FA, eds. Hematology of Infancy and Childhood, 8th ed. Philadelphia, PA: Saunders/Elsevier; 2015:1076-1102.

3. Veyradier A, Lavergne JM, Ribba AS, et al. Ten candidate ADAMTS13 mutations in six French families with congenital thrombotic thrombocytopenic purpura (Upshaw-Schulman syndrome). J Thromb Haemost. 2004;2:424 429.

4. Gussow AB, Petrovski S, Wang Q, et al. The intolerance to functional genetic variation of protein domains predicts the localization of pathogenic mutations within genes. Genome Biol. 2016;17:9.

5. Tsai HM. Physiologic cleavage of von Willebrand factor by a plasma protease is dependent on its conformation and requires calcium ion. Blood. 1996;87:4235-4244.

6. Adzhubei IA, Schmidt S, Peshkin L, et al. A method and server for predicting damaging missense mutations. Nat Methods. 2010;7:248-249.

7. Reva B, Antipin Y, Sander C. Predicting the functional impact of protein mutations: application to cancer genomics. Nucleic Acids Res. 2011;39:e118.

8. Genomes Project C, Auton A, Brooks LD, et al. A global reference for human genetic variation. Nature. 2015;526:68-74.

9. Exome Variant Server NGESPE. Available at: http://evs.gs. washington.edu/EVS/. Accessed May 30, 2019.

10. Lek M, Karczewski KJ, Minikel EV, et al. Analysis of proteincoding genetic variation in 60,706 humans. Nature. 2016;536: 285-291.

11. Richards S, Aziz N, Bale S, et al. Standards and guidelines for the interpretation of sequence variants: a joint consensus recommendation of the American College of Medical Genetics and Genomics and the Association for Molecular Pathology. Genet Med. 2015;17:405-424.

12. Peyvandi F, Mannucci PM, Valsecchi C, et al. ADAMTS13 content in plasma-derived factor VIII/von Willebrand factor concentrates. Am J Hematol. 2013;88:895-898.

13. Aledort LM, Singleton TC, Ulsh PJ. Treatment of congenital thrombotic thrombocytopenia purpura: a new paradigm. $J$ Pediatr Hematol Oncol. 2017;39:524-527.

14. Scully M, Knobl P, Kentouche $K$, et al. Recombinant ADAMTS-13: first-in-human pharmacokinetics and safety in congenital thrombotic thrombocytopenic purpura. Blood. 2017; 130:2055-2063.

15. Hereditary Thrombotic Thrombocytopenic Purpura Registry. Available at: https://ttpregistry.net.

16. Van Dorland HA, Mansouri Taleghani M, Sakai K, et al. The International Hereditary Thrombotic Thrombocytopenic Purpura Registry: key findings at enrolment until 2017. Haematologica. 2019;104:2107-2115. 